Two-dimensional echocardiography has limitations in detecting left atrial appendage thrombi and small left atrial cavity thrombi $(<1 \mathrm{~cm}), 4,5$ Transesophageal echocardiography is the best noninvasive test for detecting left atrial thrombi. In both patients, small residual left atrial thrombi could not be excluded because the latter test was not available. In fact, echocardiographic findings in patient 1 and coronary angiographic findings in patient 2 suggested residual left atrial throm$\mathrm{bi}$, and there might have been some risk of systemic embolization in performing PTMC. We thought it safe to proceed with PTMC, however, because potential contact of the balloon catheter with the likely organized thrombi in the left atrial posterior wall and appendage can be avoided when PTMC is properly executed using the Inoue balloon catheter.

Neither patient had had mitral stenosis or left atrial thrombi before. They were treated with warfarin because the incidence of systemic embolism in patients with left atrial thrombus and atrial fibrillation is higher than in patients without thrombus and in sinus rhythm. ${ }^{5}$ We do not know whether the patients' left atrial cavity thrombi resolved because of anticoagulant treatment. There are few studies regarding the natural history of left atrial thrombi in patients with rheumatic mitral valve disease. In a retrospective study of patients with rheumatic mitral valve disease and left atrial thrombus, Beppu et al ${ }^{5}$ observed that warfarin had no effect on the size of the thrombus. In their 2-dimensional echocardiographic study, the size of the thrombus was reduced in $29 \%$, unchanged in $57 \%$ and enlarged in $14 \%$ of the 28 warfarin-treated patients, while it was reduced in $19 \%$, unchanged in $62 \%$ and enlarged in $19 \%$ of the 27 untreated patients. Thrombus resolution was not observed in any of the patients. However, follow-up echocardiographic studies were made after about 1 month. If they had extended the observation period to 7 months or 1 year, as in our patients, they might have observed resolution of the left atrial thrombus in some patients. Understanding the natural history of left atrial thrombus in patients with mitral stenosis warrants a prospective study.

1. Inoue $K$, Owaki $T$, Nakamura $T$, Kitamura $F$, Miyamoto N. Clinical application of transvenous mitral commissurotomy by a new balloon catheter. $J$ Thorac Cardiovasc Sury 1984;87:394-402.

2. Inoue K, Nobuyoshi M, Chen C, Hung JS. Advantage of Inoue-balloon (self-positioning balloon) in percutaneous transvenous mitral commissurotomy (abstr). Circulation 1988. (suppl II)78:II-490.

3. McKay CR, Kawanishi DT, Kotlewski A, Parise K, Odom-Maryon T, Gonzalez A, Reid CL, Rahim toola SH. Improvement in exercise capacity and exercise hemodynamics 3 months after double-balloon, catheter balloon valvuloplasty treatment of patients with symptomatic mitral stenosis. Circulation 1988 77:1013-1021

4. Shrestha NK, Moreno FL, Narciso FV, Torres L Calleja HB. Two-dimensional echucardiographic diagnosis of left atrial thrombus in rheumatic heart disease. A clinicopathologic study. Circulation 1983. 67:341-347.

5. Beppu S, Park YD, Sakakibara H, Nagata S Nimura Y. Clinical features of intracardiac throm bosis based on echocardiographic observation. $J p n$ Circ 1984:48.75-82.

6. Colman T, de Ubago JLM, Figueroa A, Poma JL, Gallo I, Mortera C, Pajaron A, Duran CMG Coronary arteriography and atrial thrombosis in mitral valve disease. Am J Cardiol 1981,47:973-977.

\section{Transient Atrioventricular Block Resulting from Left Ventricular Angiography in Infants with Ventricular Septal Defect}

Roger P. Vermilion, MD, Robert H. Beekman, MD,

Dennis C. Crowley, MD, and Amnon Rosenthal, MD

C onduction disturbances often occur with manipulation of catheters within the atria or ventricles during cardiac catheterization. ${ }^{1,2}$ Catheter movement may induce transient right or left bundle branch block, or complete heart block in patients with or without preexistent bundle branch block. ${ }^{3-6}$ To our knowledge, however, there have been no previous reports of complete heart block resulting from an intramyocardial stain in the region of the atrio-

From the Division of Pediatric Cardiology, C.S. Mott Children's Hospital, University of Michigan Medical Center, 1500 East Medical Center Drive, Ann Arbor, Michigan 48109 . 0204. This study was supported by grant 3M01-RR00042 from the General Research Center, National Institutes of Health, Bethesda, Maryland. Manuscript received January 4, 1989; revised manuscript received April 7, 1989, and accepted April 8. ventricular (AV) conduction system. This report describes 2 infants who developed transient complete heart block following injection of contrast medium in the left ventricle. In each, complete heart block resulted from the intra-myocardial injection of contrast medium in the region of the AV conduction system.

CASE 1: The patient was an 11month-old infant weighing $6.5 \mathrm{~kg}$ with a large perimembranous ventricular septal defect (VSD). The infant was given maintenance digoxin and an electrocardiogram the day before catheterization revealed no evidence of $A V$ conduction disturbance. At catheterization, the presence of a large perimembranous $V S D$ was confirmed. A $4 F r$ pigtail catheter (Universal Medical Instrument Corp., Ballston Spa) was ret- rogradely advanced across the aortic valve into the left ventricle for a cineangiogram. A Medrad Mark IV injector was used for injection of $10 \mathrm{ml}$ iohexol at $16 \mathrm{ml} / \mathrm{s}$, pressure limit of 600 pounds/square inch and an injection rise time of zero. The injection resulted in 3 ventricular premature complexes followed by complete $A V$ block and ventricular asystole (Figure 1). After a 6-second pause, spontaneous ventricular activity began with a rate $<30$ beats/ min. Cardiopulmonary resuscitation was started immediately, and right ventricular pacing was begun. Within 3 minutes AV conduction had returned, and at 15 minutes the $P R$ interval had returned to normal. $R e$ view of the cineangiogram revealed a myocardial stain at the left superior margin of the ventricular septum along the inferior rim of the VSD (Figure 2). At operation 3 weeks later, there were no visible myocardial changes in the region of the previous stain. A postoperative electrocardiogram demonstrated no evidence of $A V$ conduction abnormality. 
CASE 2: This patient was a 14day-old infant weighing $4.2 \mathrm{~kg}$ with coarctation of the aorta and a large perimembranous VSD. Digoxin was started 2 days before the catheterization. An electrocardiogram obtained before catheterization revealed a normal $P R$ interval and no evidence of $A V$ conduction disturbance. Catheterization disclosed the presence of a discrete coarctation of the aorta and a large perimembranous VSD. A $4 F r$ pigtail catheter was retrogradely advanced across the aortic valve into the left ventricle for a cineangiogram. A Medrad Mark $I V$ injector was used for injection of $4 \mathrm{ml}$ iohexol at $10 \mathrm{ml} / \mathrm{s}$, pressure limit of 600 pounds/square inch, and an injection rise time of zero. This injection resulted immediately in complete AV block with a ventricular rate $<40$ beats/min. There was no response to atropine and epinephrine. Subsequently, isoproterenol was given with a rapid response in ventricular rate to $>100$ beats/min. By 30 minutes $A V$ conduction had returned with a normal $P R$ interval. Review of the cineangiogram revealed a myocardial stain at the left superior margin of the ventricular septum along the inferior rim of the VSD. At operation 2 days later, there were no visible myocardial changes in the region of the previous stain. A postoperative electrocardiogram revealed no evidence of $A V$ conduction abnormality.

Both infants who developed transient complete heart block during left ventricular angiography had a large perimembranous VSD and no prior electrocardiographic evidence of an AV conduction disturbance. To our knowledge, there has been no previous report of this complication of cardiac catheterization and angiog-

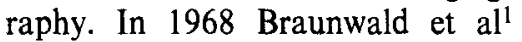
reported 1 infant who developed complete heart block following left ventricular angiography, but the cir- cumstances of the event were not described in sufficient detail to allow insight into the mechanism involved.

Complete heart block in these 2 infants almost certainly resulted from transient injury to the conduction system near the His bundle as evidenced by the myocardial stains in this region. In each child the large perimembranous VSD left the conduction tissue exposed along the inferior rim of the defect. On each injection, although the $4 \mathrm{Fr}$ pigtail catheter was initially placed near the left ventricular apex, there was recoil of the catheter away from the apex and toward the left ventricular outflow tract. Consequently the catheter side holes came to rest on the superior portion of the interventricular septum where the intramyocardial contrast injection occurred. The resulting injury to the AV conduction tissue produced AV block that required treatment, but was transient in both infants.

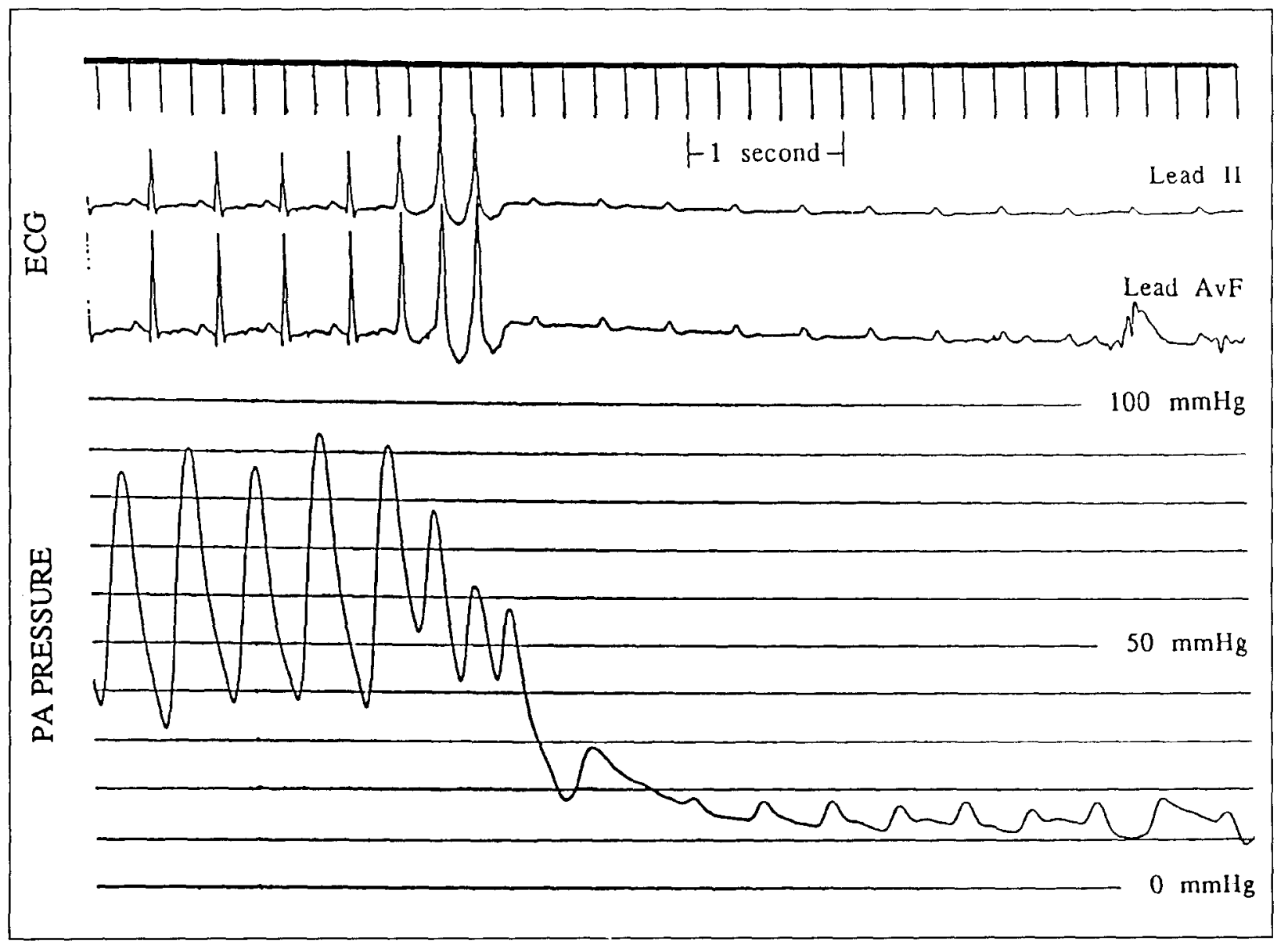

FIGURE 1. Electrocandiogram (ECG) and pumonary artery (PA) preseure recording during left ventricular angiography in patient 1. The three ventricular premature complexes mark the time of injection. Immediately afterward, complete atrioventricular block and ventricular asystole occurred. 


\section{CASE REPORTS}

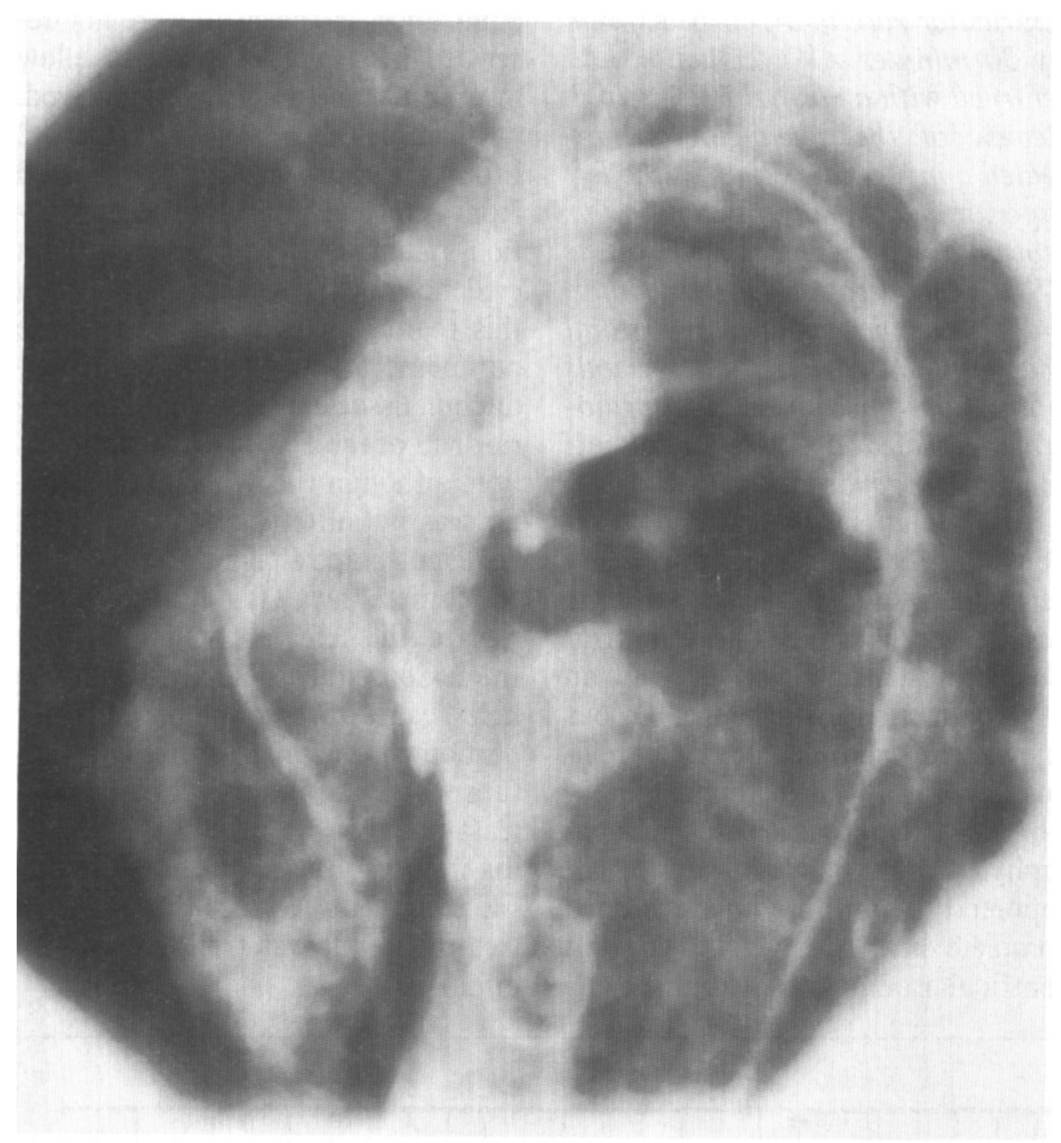

FIGURE 2. Left ventricular angiogram (long axial oblique) in patient 1 demonstrating a stain in the upper interventricular septum along the inferior margin of a perimembranous ventricular septal defect.

This report suggests that infants with a large perimembranous VSD may be at particular risk for conduction system injury from myocardial stains during left ventricular angiog-
Our experience suggests 4 potential ways to diminish the risk of conduction system injury during left ventricular angiography in infants with VSDs. First, if patient size permits, a 5Fr catheter might be used rather than the 4 Fr catheter. Second, particular care should be taken in these infants to ensure that the catheter is positioned well into the ventricle and near the apex. Third, injections using a more gradual rise time might be useful in diminishing recoil of the catheter into the left ventricular outflow tract during the injection. Fourth, modification of the catheter to move the side holes closer to the pigtail, or away from the outer rim of the pigtail, might decrease the chance of injecting contrast medium directly into the myocardium of the interventricular septum.

1. Braunwald E, Gorlin R, McIntosh HD, Ross RS, Rudolph AM, Swan HJC. Cooperative study on cardiac catheterization. Circulation 1968;(suppl III) 37:1-35.

2. Stanger P, Heymann MA, Tarnoff $H$, Hoffman JIE, Rudolph AM. Complications of cardiac catheterization of neonates, infants, and children. Circulation 1974;50:595-608.

3. Freed MD, Rosenthal A. Complete heart block after cardiac catheterization: a rare complication. Pediatrics 1973:51:935-938.

4. Goldman IR, Blount SG, Friedlich AL, Bing RJ. Electrocardiographic observations during cardiac catheterization. Bull Johns Hopkins Hosp 1950; 86:14l.

5. Gupta PK, Haft JI. Complete heart block complicating cardiac catheterization. Chest 1972,61:185187.

6. Stein PD, Mathur VS, Herman MV, Levine HD. Complete heart block induced during cardiac catheterization of patients with pre-existent bundle branch block. Circulation 1966;34:783-791. 